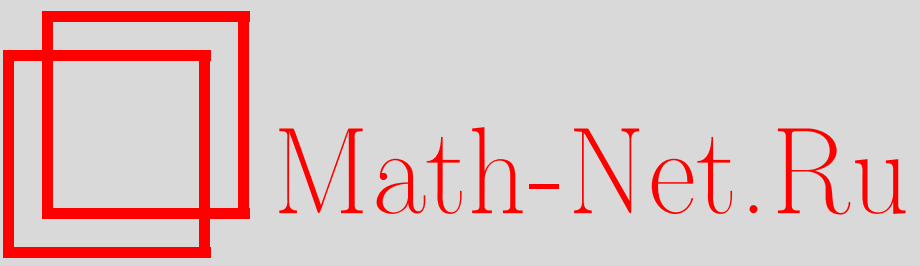

Г. Г. Козлов, Спектр поглощения одномерной цепочки с экситоном Френкеля при диагональном беспорядке в виде гиперболических дефектов, ТМФ, 1997, том 113, номер 2, 331-337

DOI: https://doi.org/10.4213/tmf1082

Использование Общероссийского математического портала Math-Net.Ru подразумевает, что вы прочитали и согласны с пользовательским соглашением

http: //www . mathnet.ru/rus/agreement

Параметры загрузки:

IP : 3.85 .5 .30

26 апреля 2023 г., 10:53:20 
ТЕОРЕТИЧЕСКАЯ

И МАТЕМАТИЧЕСКАЯ

ФИЗИКА

Том 113, № 2

ноябрь, 1997

Г. Г. Козлов*

\section{СПЕКТР ПОГЛОШЕНИЯ ОДНОМЕРНОЙ ЦЕПОЧКИ С ЭКСИТОНОМ ФРЕНКЕЛЯ ПРИ ДИАГОНАЛЬНОМ БЕСПОРЯДКЕ В ВИДЕ ГИПЕРБОЛИЧЕСКИХ ДЕФЕКТОВ}

\footnotetext{
Предложен метод вычисления спектра поглощения длинной, одномерной, замкнутой в кольцо цепочки с экситоном Френкеля при диагональном беспорядке в виде гиперболических особенностей атомного расщепления как функции положения атома. Показано, что наличие таких дефектов приводит к появлению крыла в экситонной зоне бездефектной цепочки, форма которого не зависит от числа и взаимного расположения дефектов, а величина пропорциональна сумме амплитуд дефектов. Предложенный метод использует только континуальное приближение.
}

\section{1. ВВЕДЕНИЕ}

Успехи теории трансляционно-симметричных систем в значительной степени связаны с возможностью использования аппарата функций Блоха. Переход к представлению Блоха позволяет сразу сильно упростить, а иногда и решить самые разнообразные задачи физики трансляционно-симметричных систем. В теории систем, не имеющих трансляционной симметрии, отсутствует такой универсальньй подход, что обусловливает значительные математические трудности при анализе даже простейших систем подобного типа. Одной из таких систем является модель с возбуждениями типа экситонов Френкеля - совокупность двухуровневых атомов, связанных взаимодействием, способным переносить возбуждение с одного атома на другой. Причем обычно рассматривается только одноэкситонная часть энергетического спектра, соответствуюшая наличию только одного возбужденного атома. Если атомы расположены симметрично, но имеют различное расшепление уровней, то такую систему называют диагонально разупорядоченной. Две задачи такого типа - задача Дайсона [1] и задача Ллойда [2] являются чуть ли не единственными, допускаюшими точное решение. Для таких систем разрабатывались также приближенные методы расчета (типа метода когерентного потенциала [3]). Часто критерием качества таких методов является сравнение с машинным экспериментом, причем обычно неясно, почему приближенный расчет согласуется (если согласуется) с численным. Поэтому с точки зрения автора представляют интерес даже отвлеченные модели трансляционно-несимметричных систем, для которых можно

*ВНЦ ГОИ им. С. И. Вавилова, Санкт-Петербург, Россия 
предложить достаточно убедительно с математической точки зрения схему решения. В настояшей статье делается такая попытка.

Рассматривается задача об экситоне Френкеля в бесконечно длинной, замкнутой в кольцо цепочке двухуровневых атомов, причем межатомное взаимодействие и энергия атомного расщепления как функция координаты атома имеют соответственно вид

$$
\begin{aligned}
w(z) & =V \exp \left(-\left|\frac{z}{R}\right|\right), \\
\epsilon(z) & =\sum_{r}^{N} \frac{a_{r}}{\left(z-R_{r}\right)}, \quad a_{r}>0,
\end{aligned}
$$

т.е. введен диагональный беспорядок в виде произвольного числа $N$ произвольно расположенных в точках $R_{r}$ гиперболических особенностей (ниже гиперболические дефекты) с положительными (знакопостоянными) амплитудами $a_{r}$. В данной работе предлагается метод вычисления спектра поглошения такой цепочки при нулевой температуре. Известно, что спектр поглощения бездефектной цепочки представляет собой синглет, поэтому изменения спектра, связанные с дефектами, более заметны, чем изменения плотности состояний. В работе показано, что наличие гиперболических дефектов приводит к появлению крыла в экситонной зоне бездефектной цепочки, причем его форма не зависит от числа и взаимного расположения дефектов, а величина пропорциональна сумме амплитуд дефектов $\sum_{r}^{N} a_{r}$. Этот результат представляется несколько неожиданным. При расчете используется метод, предложенный в [4], использующий только одно приближение - континуальное (т.е. замену решеточных сумм интегралами). Эффективность такого подхода обоснована в [4].

\section{2. СХЕМА МЕТОДА}

Ниже для вычисления спектра поглощения цепочки, описанной во введении, мы будем пользоваться методом, предложенным в [4]. Приведем здесь полученные там результаты. Если каждую линию спектра считать лоренцевой с шириной $\delta$, то спектр поглощения $A(\Omega)$ цепочки с параметрами типа $(1),(2)$ и координатами концов $L$ и $-L$ можно рассчитать по формуле

$$
A(\Omega)=-\frac{1}{\pi} \operatorname{Im} \int_{-L}^{L} \frac{\rho \Psi(z) d z}{E-\epsilon(z)},
$$

где $\Psi(z)$ определяется из уравнения

$$
\frac{d^{2} \Psi}{d z^{2}}+\left(\frac{1}{R}\right)^{2}\left(\frac{W}{E-\epsilon(z)}-1\right) \Psi=-\left(\frac{1}{R}\right)^{2} .
$$

Здесь $R$ - радиус взаимодействия (1), $W=2 \rho V R$ - ширина экситонной зоны бездефектной цепочки, $\rho$ - плотность атомов в цепочке, $\epsilon(z)$ - рельеф атомного расщепления, $\Omega$ - энергия кванта падаюшего света, $E=\Omega+i \delta, \delta>0$. Для замкнутой в кольцо цепочки, которую мы рассматриваем, функция $\Psi(z)$ должна удовлетворять условиям цикличности

$$
\begin{aligned}
\Psi(L) & =\Psi(-L), \\
d \Psi(L) / d z & =d \Psi(-L) / d z .
\end{aligned}
$$


Интегрируя уравнение (4) по $z$ от $-L$ до $L$ и принимая во внимание (5) и (6), мы убедимся в том, что интеграл в (3) может быть выражен следующим образом:

$$
2 V R \int_{-L}^{L} \frac{\rho \Psi(z) d z}{E-\epsilon(z)}=\int_{-L}^{L}(\Psi(z)-1) d z .
$$

Таким образом, задача сводится к вычислению интеграла $\int_{-L}^{L} \Psi(z) d z$. Идея заключается в том, чтобы вычислять этот интеграл не по вешественной оси, а по полукругу радиуса $L$ в верхней полуплоскости комплексного переменного $z$. Это можно сделать в том случае, если $\Psi(z)$ не имеет особенностей в верхней полуплоскости (полюсов, точек ветвления), за чем необходимо проследить. Далее, если $\epsilon(z)$ убывает на указанном полукруге, то уравнение (4) может быть решено на полукруге по теории возмущений тем более точно, чем больше радиус полукруга (длина цепочки), и при $L \rightarrow \infty$ ошибка будет стремиться к нулю. Известно [5], что решение уравнения (4) не будет иметь особенностей в верхней полуплоскости комплексного $z$ в том случае, если функция $W /(E-\epsilon(z))$ там однозначна и не имеет полюсов. Покажем, что это так для $\epsilon(z)$ вида $(2)$ в случае, когда $E$ имеет малую положительную мнимую часть. Если $E$ вешественна, то уравнение $E-\epsilon(z)=0$ имеет $N$ вещественных корней $z_{k}(E), k=1, \ldots, N$. Если $E$ получает малую мнимую добавку $i \delta, \delta>0$, корни получают прирашение

$$
\delta z_{k}=\frac{i \delta}{d \epsilon\left(z_{k}\right) / d z} .
$$

Так как $\epsilon(z)$ из формулы (2) - монотонно убывающая функция, все указанные корни сдвинутся в нижнюю полуплоскость и, следовательно, $W /(E-\epsilon(z))$ в нашем случае не имеет полюсов в верхней полуплоскости. В следующем разделе мы проведем расчет спектра по описанной выше схеме.

\section{PACЧET CПEKTPA}

Перепишем (4) в виде

$$
\Psi^{\prime \prime}+q^{2} \Psi+\Delta(z) \Psi=-(1 / R)^{2}
$$

где

$$
\begin{aligned}
q^{2} & \equiv\left(\frac{1}{R}\right)^{2}\left(\frac{W}{E}-1\right), \\
\Delta(z) & \equiv\left(\frac{1}{R}\right)^{2} \frac{W \epsilon(z)}{[E-\epsilon(z)] E} .
\end{aligned}
$$

Здесь $q$ - волновой вектор экситона с энергией $E$ в бездефектной цепочке, функция $\Delta(z)$ описывает дефекты и убывает на полукруге радиуса $L$ (ниже будем называть его большим полукругом) в верхней полуплоскости комплексного $z$. Найдем решение (8) на большом полукруге в первом приближении по $\Delta(z)$. Ишем решение (8) в виде $\Psi(z)=\Psi_{0}(z)+\Psi_{1}(z)+\cdots$, где нижний индекс указывает порядок поправки по $\Delta(z)$. Эти поправки удовлетворяют уравнениям

$$
\begin{aligned}
& \Psi_{0}^{\prime \prime}+q^{2} \Psi_{0}=-(1 / R)^{2} \\
& \Psi_{1}^{\prime \prime}+q^{2} \Psi_{1}=-\Delta \Psi_{0} .
\end{aligned}
$$


Решение каждого из этих уравнений не вызывает затруднений, и $\Psi(z)$ в рассматриваемом приближении имеет вид

$$
\begin{aligned}
\Psi(z)= & -\left(\frac{1}{q R}\right)^{2}+\left(\frac{1}{q R}\right)^{2} \int_{L}^{z} \frac{\Delta(\xi)}{q} \sin [q(z-\xi)] d \xi+ \\
& +C_{+}\left(e^{i q z}-\int_{L}^{z} \frac{\Delta(\xi)}{q} e^{i q \xi} \sin [q(z-\xi)] d \xi\right)+ \\
& +C_{-}\left(e^{-i q z}-\int_{L}^{z} \frac{\Delta(\xi)}{q} e^{-i q \xi} \sin [q(z-\xi)] d \xi\right)
\end{aligned}
$$

Все интегрирования идут от правого конца цепочки по большому полукругу, и, т.к. $\Delta(z)$ там мало (при $L \rightarrow \infty$ ), мы ожидаем, что решение (13) на большом полукруге практически совпадает с точным. Для определения констант $C_{+}, C_{-}$используем полученное решение (13) и условия (5), (6), что возможно, т.к. точки $\pm L$ лежат на большом полукруге, где полученное решение справедливо. В результате имеем следуюшую систему уравнений для $C_{+}$и $C_{-}$:

$$
\begin{aligned}
C_{+}\left\{I_{c}(q, q)-2 q \sin (q L)\right\}+C_{-}\left\{I_{c}(-q, q)-2 q \sin (q L)\right\} & =I_{c}(0, q) /(R q)^{2}, \\
C_{+}\left\{i I_{s}(q, q)-2 q \sin (q L)\right\}+C_{-}\left\{i I_{s}(-q, q)+2 q \sin (q L)\right\} & =i I_{s}(0, q) /(R q)^{2},
\end{aligned}
$$

где

$$
\begin{aligned}
& I_{c}(\alpha, \beta) \equiv \int_{L}^{-L} \Delta(\xi) e^{i \alpha \xi} \cos [\beta(L+\xi)] d \xi \\
& I_{s}(\alpha, \beta) \equiv-\int_{L}^{-L} \Delta(\xi) e^{i \alpha \xi} \sin [\beta(L+\xi)] d \xi
\end{aligned}
$$

Напомним, что все интегрирования ведутся по большому полукругу.

Будем считать $q$ вещественным и положительным. Это означает, что әнергия $E$ nопадает в әкситонную зону бездефектной решетки. Займемся вычислением интегралов типа (16), (17), входящих в (14), (15). При этом мы будем всюду, где это возможно, переходить к пределу $L \rightarrow \infty$. Поясним, например, как вычисляется

$$
I_{s}(0, q)=-\int_{L}^{-L} d \xi \Delta(\xi) \sin [q(L+\xi)]
$$

На большом полукруге в синусе следует оставить только растушую экспоненту $\exp [-i q(L+\xi)]$. В то же время эта экспонента стремится к нулю на нижнем большом полукруге, поэтому

$$
\int_{L}^{-L} \Delta(\xi) \exp [-i q(L+\xi)] d \xi
$$

можно вычислять по всему большому кругу, что нетрудно сделать с помошњю вычетов. 
Приведем результаты вычислений интегралов, входящих в (14), (15):

$$
\begin{aligned}
& I_{s}(0, q)=\pi e^{-i q L} \sum_{\xi} \operatorname{Res}\left[\Delta(\xi) e^{-i q \xi}\right], \\
& I_{s}(q, q)=\frac{1}{2 i} e^{-i q L} \int_{L}^{-L} \Delta(\xi) d \xi \\
& I_{c}(0, q)=\pi i e^{-i q L} \sum_{\xi} \operatorname{Res}\left[\Delta(\xi) e^{-i q \xi}\right], \\
& I_{c}(q, q)=\frac{1}{2} e^{-i q L} \int_{L}^{-L} \Delta(\xi) d \xi .
\end{aligned}
$$

Так как $I_{c}(0, q)=i I_{s}(0, q)$ и $i I_{s}(q, q)=I_{c}(q, q)$, то $C_{-}=0$ и остальные интегралы из $(14),(15)$ нам не понадобятся. Для $C_{+}$получаем следуюшую формулу:

$$
C_{+}=\left(\frac{1}{R q}\right)^{2} \frac{2 \pi i e^{-i q L} \sum_{\xi} \operatorname{Res}\left[\Delta(\xi) e^{-i q \xi}\right]}{e^{-i q L} \int_{L}^{-L} \Delta(\xi) d \xi-4 q \sin (q L)}
$$

Для окончательного вычисления $\int_{L}^{-L}(\Psi(z)-1) d z$ нам еше потребуются интегралы (см. формулу (13))

$$
\begin{gathered}
\int_{L}^{-L} d z \int_{L}^{z} d \xi \Delta(\xi) \sin [q(z-\xi)]=-\frac{i \pi}{q} e^{-i q L} \sum_{\xi} \operatorname{Res}\left[\Delta(\xi) e^{-i q \xi}\right]+\frac{1}{q} \int_{L}^{-L} \Delta(z) d z \\
\int_{L}^{-L} d z \int_{L}^{z} d \xi \Delta(\xi) e^{i q \xi} \sin [q(z-\xi)]=-\frac{e^{-i q L}}{2 q} \int_{L}^{-L} \Delta(z) d z
\end{gathered}
$$

Эти выражения получаются разложением синуса на экспоненты, интегрированием по частям, сохранением только растуших на большом полукруге экспонент и переходом к интегрированию по большому кругу, как при получении выражений (18)-(21). Принимая во внимание, что

$$
\int_{L}^{-L} \frac{\epsilon(z) d z}{E-\epsilon(z)}=\frac{i \pi}{E} \sum_{r} a_{r}
$$

(интегрирование идет по большому полукругу), уже нетрудно получить для спектра поглошения следуюшую окончательную формулу:

$$
A(\Omega)=-\frac{1}{\pi} \operatorname{Im}\left(\frac{2 L \rho}{E-W}-i \pi \rho\left(\frac{1}{E-W}\right)^{2} \sum_{r} a_{r}\right), \quad E=\Omega+i \delta
$$

\section{4. ОБСУЖДДЕНИЕ}

Первое слагаемое в (23) дает синглет и не зависит от наличия дефектов. Второе слагаемое дает крыло в экситонной зоне. Несколько неожиданным представляется независимость его формы от взаимного положения $R_{r}$ дефектов. Что касается амплитуды крыла, то она оказывается пропорциональной сумме амплитуд дефектов. Приведенный расчет имеет “физический” уровень строгости. Для строгого расчета следует написать весь ряд теории возмушений по $\Delta(z)$ для решения уравнения (4) и убедиться в том, что 
влияние всех членов этого ряда, кроме рассмотренного выше члена первого порядка, на большом полукруге стремится к нулю при $L \rightarrow \infty$. Это было проделано, причем оказалось, что результат (23) является верным только при вещественном $q$, т.е. при энергии, попадающей в экситонную зону бездефектной цепочки. В противном случае вкладом членов порядка выше первого пренебречь нельзя. Такое поведение следует обсудить. Как показано в [4], $\Psi(z)$ связана с функцией Грина системы, и, следовательно, решение (23) можно было бы аналитически продолжить в область энергий, не попадающих в экситонную зону бездефектной цепочки. Это было бы корректно, если бы мы имели дело с точной функцией Грина, однако уравнение (4) получено в [4] с использованием континуального приближения, поэтому (23) может давать заметные отклонения от точного спектра вне экситонной зоны, где приведенный в предыдущем разделе расчет несправедлив. Проведенный компьютерный анализ подтверждает это замечание.

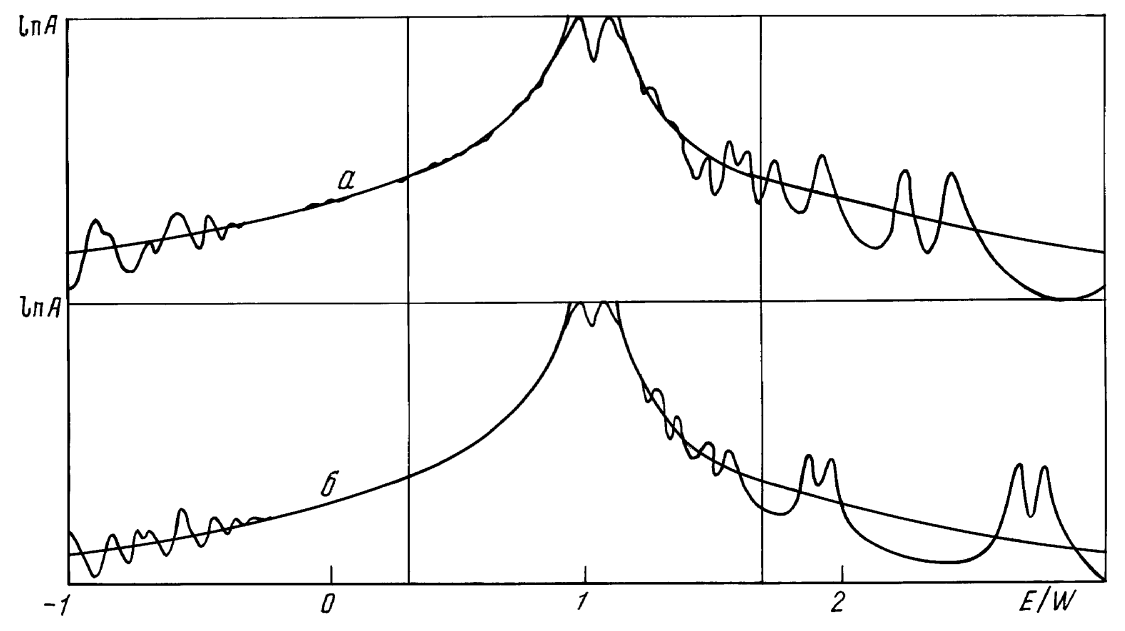

На рисунке приведены логарифмические спектры поглощения двух цепочек с различным количеством, расположением и амплитудой гиперболических дефектов при $W=1$, $\rho=1, \delta=0.02, R=2$. Число частиц во всех случаях 600 , т.е. $2 L=600$. На верхней кривой $a$ изображен логарифмический спектр поглощения цепочки с пятью гиперболическими дефектами. Их положения (номера узлов, на которых атомное расшепление обрашается в бесконечность, т.е. эти атомы эффективно отсутствуют) таковы: 200, 250, 300, 350, 400. Амплитуды дефектов - соответственно 2, 3, 3, 6, 1. На нижней кривой 6 изображено то же для цепочки с двумя дефектами, их амплитуды -5 и 5 , положения -250 и 350 . Для каждого численного спектра приведен спектр, рассчитанный по формуле (23) (сглаженные кривые), причем никакой подгонки (ни по амплитуде, ни по форме) не делалось. Энергия отложена в единицах $W$. Из рисунка видно, что при $0<\Omega<1$ (экситонная зона) численные спектры практически полностью совпадают с теоретическими, причем форма спектров не зависит от величины и расположения дефектов. Расхождение при $\Omega \approx 1$ объясняется конечностью цепочки, т.к. в этой области энергий величина $q^{2}\left(q^{2} \approx 0\right)$ может быть сравнима с $\Delta(z)$, которая конечна на большом круге вследствие конечности цепочки. Вне экситонной зоны можно говорить лиш о соответствии "в среднем" (его можно существенно улучшить, увеличив мнимую часть 
энергии $\delta$ ). В этой области спектр зависит от расположения и величины дефектов. Возможно, предложенный подход удастся применить к более реалистическим моделям дефектных цепочек.

Работа выполнена при поддержке РФФИ, грант 95-03-09221а.

\section{Список литературы}

[1] F. Dyson. Phys. Rev. 1953. V. 92. P. 1331.

[2] P. Lloyd. J. Phys. C: Solid State Phys. 1969. V. 2. P. 1717.

[3] И. М. Лифиии, С. А. Гредескул, Л. А. Пастур. Введение в теорию разупорядоченных систем. М.: Наука, 1982.

[4] Г. Г. Козлов. Оптика и спектроскопия. 1997. Т. 82. № 2. С. 266-271.

[5] В. И. Смирнов. Курс высшей математики. Т. 3. М.: Наука, 1974. С.347.

Поступила в редакцию 6.V.1997 г.

\section{G. G. Kozlov}

\section{ABSORPTION SPECTRUM OF ONE-DIMENSIONAL CHAIN WITH FRENKEL'S EXITON UNDER DIAGONAL DISORDER REPRESENTED BY HYPERBOLIC DEFECTS}

A method for calculating the absorption spectrum of a long one-dimensional closed in a ring chain with Frenkel's exiton under diagonal disorder represented by hyperbolic singularities of atomic splitting as a function of atomic absorption is proposed. It is shown that such defects lead to appearance of a wing in a exiton zone of chain without defects, whose form does not depend on number and mutual positions of defects, while it's value is proportional to the sum of amplitudes of defects. The proposed method uses a continual approximation only. 